\title{
Psicopatología en familiares de tres generaciones: un estudio epidemiológico en la Ciudad de México
}

Jorge J. C araveo-Anduaga, MC, MSP, (1) Humberto N icolini-Sánchez, MC, Dr en CM, (1) Antonio Villa-Romero, MC, M en $C,{ }^{(2)}$ Fernando A.W agner, MPH, SCD. ${ }^{(3)}$

\section{Caraveo-Anduaga JJ, Nicolini-Sánchez H, Villa-Romero A, Wagner FA. Psicopatología en familiares de tres generaciones: un estudio epidemiológico en la Ciudad de México. Salud Publica Mex 2005;47:23-29. El texto completo en inglés de este artículo está disponible en: http://www.insp.mx/salud/47/eng}

\section{Resumen}

Objetivo. Estimar el riesgo para desarrollar psicopatología entre padres e hijos a lo largo de tres generaciones. Ma terial y métodos. Se utilizó el método del "estudio de la historia familiar". Para el estudio se utilizaron datos de una encuesta probabilística de hogares efectuada en 1995 en la Ciudad de México. Los probandos fueron adultos entre los 18 a 65 años de edad ( $n=1$ 932) a quienes se entrevistó utilizando la Entrevista Internacional Diagnóstica Compuesta. Además, se obtuvo información acerca de antecedentes psiquiátricos en sus padres empleando el método y los criterios diagnósticos de la historia familiar. Finalmente, en aquellos adultos con hijos de entre 4 a 16 años viviendo en el mismo hogar $(n=925)$ se les aplicó un cuestionario para detectar la presencia de psicopatología en cada uno de sus hijos, obteniéndose informacion acerca de 1686 niños y adolescentes. Para estimar el riesgo de morbilidad entre generaciones y la interacción entre éstas, se distinguió cuando sólo hubiera antecedentes en los abuelos, so lamente en los padres y cuando estaban presentes en los abuelos y en los padres. La probabilidad de ser "caso" se obtuvo a partir de la razón de momios resultante de los análisis de regresión logística siguiendo tres diferentes modelos: el clásico, el con efectos aleatorios y el con ecuaciones de estimación gener alizada con varianza robusta. Resultados La probabilidad de presentar psicopatología

\author{
Caraveo-Anduaga JJ, Nicolini-Sánchez H, \\ Villa-Romero A, Wagner FA. \\ Psychopathology across three family generations: \\ an epidemiological study in Mexico City. \\ Salud Publica Mex 2005;47:23-29. \\ The English version of this paper \\ is available at: http://www.insp. $\mathrm{mx} / \mathrm{salud} / \mathbf{4 7 / e n g}$
}

\section{A bstract}

Objective. To estimate the risk of psychiatric disorders across three family generations. Material and Methods. The research design corresponds to a "family history stu$d y^{\prime \prime}$ and is based upon a household survey conducted in 1995, on a representative sample of Mexico City's adult population aged 18 - 65 years $(n=1932)$. Briefly, a standardized assessment of adults' lifetime prevalence of psychiatric disorders was obtained using an amended version of the Composite International Diagnostic Interview, C ID I. In addition, all respondents provided information on their parents' psychiatric history of anxiety, affective disorders, and substance-use disorders. Also, respondents who had 4-16 year old children living in the same household were interviewed using a standardized questionnaire that included specific questions on their children's psychiatric symptoms ( $n=925$ parents, corresponding to 1686 children and adolescents). Familial psycho pathology across generations was defined as follows: a) history of psychiatric disorders only in grandparents; b) history of psychiatric disorders in one parent only (mother or father); and, c) history of psychiatric disorders history in grandparents and in parent. The risk of psychiatric morbidity was estimated via odds ratios obtained from logistic regression models with and without random-effects and using Generalized Estimation Equations with robust variance estimation. Results The risk for psychopatholo-

Esta investigación fue financiada por el Consejo N acional de Ciencia yTecnología, proyecto número 2077-H 9302 y por el N ational Institute of D rugA buse, reconocimiento número 5ROIDA11121.

(1) Instituto $\mathrm{N}$ acional de Psiquiatría Ramón de la Fuente, México, DF, México.

(2) Instituto $\mathrm{N}$ acional de Ciencias Médicas y $\mathrm{N}$ utrición Salvador Zubirán, México, DF, México.

(3) Morgan State University, Johns Hopkins University, Baltimore (MD), USA.

Fecha de recibido: 7 de enero de 2004 - Fecha de aprobado: 8 de diciembre de 2004

Solicitud de sobretiros: D r. Jorge J Caraveo Anduaga, Instituto N acional de Psiquiatría Ramón de la Fuente.

División de Investigaciones Epidemiológicas y Psicosociales. Departamento de investigaciones en servicios de salud.A venida México-Xochimilco 101, colonia San Lorenzo Huipulco, Tlalpan, 14370 México, DF, México.

Correo electrónico: caraveoj@ imp.edu.mx 
es entre dos a tres veces mayor cuando hay trastornos psiquiátricos en los padres. La probabilidad es mayor cuando existe comorbilidad entre diferentes tipos de trastornos. Conclusiones El riesgo para desarrollar psicopatología entre las generaciones es al menos moderado y relativamente estable. Los resultados sugieren que, en general, la transmisión de psicopatología entre las generaciones es acorde con la de un modelo genético mixto. El texto completo en inglés de este artículo está disponible en: http:// www.insp.mx/salud/47/eng

Palabras clave: agregación familiar ; historia familiar; psico patología; epidemiología; comorbilidad; México gy in the offspring across generations was estimated to be two to three times higher when psychiatric disorders occurred among the parents. The risk of psychiatric disorders was estimated to be higher when comorbidity among different types of disorders was present. Conclusions.The results suggest that the risk for developing psychopathology across generations is at least moderate and relatively stable. Familial transmission of psychopathology across three generations seems to resemble a genetic mixed model.The English version of this paper is available at: http:// www.insp.mx/salud/47/eng

Key words: familial aggregation; family history; psychopathology; epidemiology; comorbidity; M exico
D iversos estudios han señalado que existe una base familiar para diferentes trastornos psiquiátricos. ${ }^{1,2}$ Sin embargo, el conocimiento acerca de la agregación familiar de los trastornos psiquiátricos y del uso de sustancias está basado, principalmente, en muestras atípicas de personas afectadas, dado que la mayoría de los estudios parten de personas que han acudido a recibir atención en algún servicio médico, ${ }^{3}$ y, para la mayoría de los trastornos psiquiátricos, sólo una minoría de personas afectadas acude a los servicios de salud especializados. ${ }^{4,5}$ Además, la mayoría de los estudios familiares acerca de los trastornos psiquiátricos se han enfocado a un solo trastorno a la vez, dificultando la detección de patrones de transmisión familiar a través de diversos tipos de trastornos. ${ }^{6}$

Todos los trastornos psiquiátricos para los que se sospecha una base genética se clasifican en los llamados "trastornos complejos", esto es, aquellos que muestran evidencias de contener efectos genéticos, pero no muestran patrones de herencia monogénica. Estos trastornos tienden a resultar de la interacción entre la susceptibilidad genética y los eventos del medio ambiente. Por lo tanto, los intereses de los epidemiólogos y de los genetistas confluyen, y la necesidad de una metodología común se ha hecho evidente. ${ }^{7}$

La primera tarea en el estudio epidemiológico genético de un trastorno de tipo complejo es establecer si hay agregación familiar. Esta se define como la ocurrencia de un trastorno con una frecuencia mayor en los familiares de las personas afectadas, en comparación con la que ocurre en la población general. De acuerdo a la forma de obtener la información se distinguen dos tipos de estudios: a) aquéllos en los cuales se entrevista directamente a los familiares, denominados "estudio familiar", y b) aquéllos en los que se obtiene información a partir del probando o de otro familiar acerca de otros familiares, denominados "estudio de la historia familiar", y que ha sido el más frecuentemente utilizado y el que elegimos para el presente estudio. $^{2}$

Los estudios familiares de tipo epidemiológico pueden aportar evidencia que sugieran agregación familiar de una condición cuando: a) en los familiares de los "casos" haya mayor prevalencia de la condición bajo estudio, en comparación con la de la población de la cual proviene el "caso"; b) la prevalencia de la condición es mayor entre los familiares de los "casos" que entre los familiares de los controles, y c) la distribución de un trastorno al interior de las familias está de acuerdo con un patrón genético conocido, demostrado por análisis de segregación.

Los estudios en este campo han sugerido que existe una predisposición familiar "neurótica" cuya expresión, en términos de trastornos específicos, es variable. Esto es, diversos trastornos parecen comportarse como grupos antes que como entidades únicas con factores etiológicos y factores de riesgo diferentes. ${ }^{8,9}$ Los estudios familiares pueden ayudar a identificar subgrupos homogéneos y reducir así la heterogeneidad.

Cuando los estudios familiares han brindado evidencia de agregación familiar, surge la pregunta acerca de la contribución relativa de los genes y del ambiente. Sin embargo, ésta no será resuelta a través de esta metodología, sino por métodos que midan efectos genéticos.

Hasta el momento, en México no hay estudios epidemiológicos de tipo familiar acerca de los trastornos psiquiátricos más comunes, a pesar de existir un grupo de investigadores dedicados al campo de la biología molecular de algunos de éstos. ${ }^{10-13}$ Por lo anterior, consideramos importante examinar la agregación familiar de una gama amplia de trastornos psiquiátricos a partir de una muestra epidemiológica en nuestro medio. 
Además, una de las características distintivas y originales de este trabajo es que los estudios epidemiológicos acerca de trastornos psiquiátricos y problemas de salud mental en la literatura no han abordado conjuntamente la investigación de la presencia de patología psiquiátrica, tanto en los adultos como en los hijos de éstos. Hasta donde sabemos, ningún estudio epidemiológico en población general en la literatura mundial ha investigado el riesgo para desarrollar psicopatología en familiares de tres generaciones: abuelos, padres e hijos. En este trabajo, consideramos la presencia de piscopatología cuando en el probando hubiera algún trastorno psiquiátrico a lo largo de la vida; cuando se hubiesen reportado antecedentes de probables trastornos psiquiátricos en los padres del probando, y cuando se reportara sintomatología suficiente como para sospechar la presencia de algún trastorno en los hijos, según se detalla más adelante.

El objetivo de este trabajo es: estimar el riesgo para desarrollar psicopatología entre padres e hijos en tres generaciones. Las hipótesis son: a) la presencia de algún tipo de psicopatología es mayor en los hijos de los "casos", en comparación con los hijos de personas sin trastornos psiquiátricos, y b) a mayor psicopatología, expresada en términos de la comorbilidad, mayor es la probabilidad de que existan trastornos en miembros de generaciones subsecuentes.

\section{Material y métodos}

La información procede de una encuesta de hogares en el Distrito Federal, realizada en 1995. El diseño del muestreo fue polietápico y estratificado, teniendo como última unidad de selección a un adulto de 18 a 65 años de edad, sin remplazo, en cada hogar. El procedimiento ya ha sido descrito con mayor amplitud en otras publicaciones. ${ }^{14-16}$ La tasa de respuesta fue de $60.4 \%$ logrando 1932 entrevistas completas. Sin embargo, sólo $8 \%$ rehusó directamente participar en la encuesta.

Para cada adulto (probando), se investigó la prevalencia de trastornos psiquiátricos a lo largo de la vida empleando la Entrevista Internacional Diagnóstica Compuesta, (CIDI, por sus siglas en inglés). 5,17 También se obtuvo información acerca de la presencia de antecedentes respecto a trastornos depresivos, de ansiedad y de abuso de sustancias en sus padres (en lo sucesivo Gen 1), empleando el método y los criterios diagnósticos de la historia familiar. ${ }^{6,18,19}$ Brevemente, respecto de cada figura parental se preguntó: ¿Su (padre, madre) tuvo alguna vez un periodo de dos o más semanas en el que se encontrara, o se notara deprimido, triste o abatido?, y isu (padre, madre) tuvo periodos de un mes o más en los que constantemente estuviera nervioso, tenso o ansioso?

En caso de una respuesta afirmativa, adicionalmente, se preguntó si recibió tratamiento, fue hospitalizado, o interfirió mucho con su vida o con sus actividades, y si intentó o amenazó con suicidarse, a manera de criterios de severidad, para considerar como positivo al antecedente.

Otra información acerca de la Gen 1 incluyó preguntas sobre el uso de alcohol y otras drogas, y problemas asociados (por ejemplo, legales, de salud, familiares, sociales y laborales).

Finalmente, en aquellos adultos con hijos de entre 4 a 16 años viviendo en el mismo hogar $(n=925)$, se aplicó un cuestionario para detectar la presencia de psicopatología en cada uno de sus hijos (en lo sucesivo Gen 3), obteniéndose información acerca de 1686 niños y adolescentes. La confiabilidad interna de este cuestionario fue estimada con un coeficiente de $\mathrm{Ku}$ der-Richardson de $0.81 .^{15}$

La prevalencia de psicopatología a lo largo de la vida de los adultos (probandos) y la de sus padres, se definió de manera general respecto a la presencia o ausencia de trastornos, de acuerdo con los criterios diagnósticos de la Estadística Internacional de Enfermedades y Problemas Relacionados con la Salud (CIE-10), ${ }^{20}$ y de manera específica se agrupó por tipos de trastornos: ansiedad, afectivos, sustancias, comorbilidad ansiedadafectivos y comorbilidad de los tres tipos.

La psicopatología en los niños puede entenderse como un desarrollo normal que se ha vuelto inapropiado o desviado. Esto quiere decir, que hay conductas que puedan esperarse aparezcan en ciertas etapas del desarrollo, pero que si persisten pueden convertirse en patológicas. Cuando algunos rasgos, signos o conductas son especialmente llamativos y se presentan de manera conjunta y frecuente, convencionalmente se habla de síndromes. ${ }^{21}$

En virtud de que las conductas incluidas en el instrumento se refieren a motivos frecuentes de consulta en los servicios clínicos, se esperaba que para la mayoría de los niños se reportaran como ausentes, o bien como síntomas aislados. La distribución de las respuestas obtenidas mostró que no se reportaron síntomas para $49 \%$ de los niños y adolescentes. De hecho, la mediana fue de un síntoma por niño o adolescente; el valor correspondiente al percentil 80 fue de tres síntomas, el del percentil 90 fue de cinco síntomas, y el valor correspondiente al percentil 95 fue de siete síntomas. A partir del percentil $90,45 \%$ de los padres percibieron la necesidad de atención para sus hijos y en un estudio en el ámbito de los servicios de salud men- 
una asociación significativa con la presencia de probables trastornos en sus hijos (Gen 3). La fuerza de asociación fue mayor cuando en los probandos había comorbilidad de trastornos afectivos y de ansiedad, seguida por aquellos donde solamente hubo trastornos de ansiedad y de aquellos con los tres tipos de trastornos.

El cuadro III ofrece información acerca de la asociación entre la presencia de antecedentes psiquiátricos entre la Gen 1 y la Gen 2, por grupos de trastornos. Así, se muestra un exceso de riesgo entre los probandos cuyos padres tuvieron algún tipo de trastorno psiquiátrico especialmente ante la presencia de dos o más tipos de trastornos.

En conjunto, estos resultados sustentan la hipótesis de que a mayor severidad, expresada ésta en términos de la comorbiliad, es mayor la probabilidad de que se presente psicopatología en los descendientes.

En el siguiente modelo se evaluó el riesgo para la presencia de psicopatología en los niños y adolescentes (Gen 3) considerando la interacción en relación con el reporte: a) cuando sólo había antecedentes psicopatológicos en los abuelos, pero no en los padres de los menores; b) cuando sólo los había en el padre o madre (probandos), pero no en los abuelos, y c) cuando había antecedentes psicopatológicos tanto en los abuelos como en el probando. En el cuadro IV se aprecia que los momios de psicopatología en la Gen 3 son mayores para niños y adolescentes cuyas familias tienen antecedentes de psicopatología. La fuerza de asociación es mucho mayor cuando hay antecedentes familiares en las dos generaciones anteriores, por encima de lo que

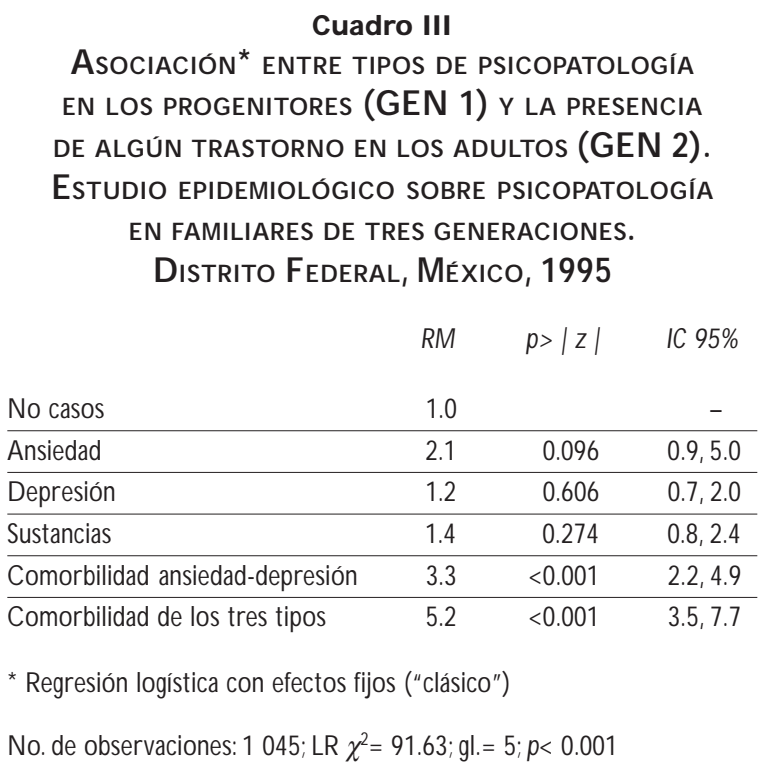

\section{Cuadro IV \\ Asociación* entre anteCedentes de PSicopatología FAMILIAR (GEN 1 Y 2) Y PSICOPATOLOGÍA EN LOS NIÑOS Y ADOLESCENTES (GEE). \\ ESTUDIO EPIDEMIOLÓGICO SOBRE PSICOPATOLOGÍA EN FAMILIARES DE TRES GENERACIONES. Distrito Federal, México, 1995}

\begin{tabular}{lccc} 
Antecedentes & RM & $p>|z|$ & IC 95\% \\
N o casos & 1.0 & & - \\
\hline Sólo en abuelos & 3.0 & 0.002 & $1.5,5.8$ \\
\hline Sólo en el padre o madre & 2.4 & 0.067 & $0.9,6.0$ \\
\hline En ambos & 7.8 & $<0.001$ & $3.9,15.4$ \\
*Regresión logística con ecuaciones de estimación generalizada
\end{tabular}

N o. de observaciones: $739 ; \mathrm{N}$ o. de grupos: $419 ; \mathrm{W}$ ald $\chi^{2}=35.26 ; \mathrm{gl} .=3 ; p<$ 0.001

podría esperarse si la combinación resultase debido al azar.

\section{Discusión}

Los resultados de este trabajo indican que, de acuerdo con lo esperado, en la población estudiada hubo un riesgo estadísticamente significativo entre la presencia de psicopatología en los progenitores y el desarrollo de trastornos psiquiátricos en los descendientes. Además, los resultados sugieren que, de manera general, el riesgo para desarrollar psicopatología entre las generaciones, es al menos moderado y relativamente estable en el sentido de que la fuerza de la asociación se mantiene de generación en generación. De manera específica, la presencia de comorbilidad entre los tipos de trastornos investigados se asocia con una mayor probabilidad para que en los descendientes se presente psicopatología. Finalmente, los resultados sugieren que, en general, la transmisión de psicopatología entre las generaciones es acorde con la de un modelo mixto.

Como se señaló en la introducción, no hay antecedentes en la literatura mexicana acerca de estudios de epidemiología psiquiátrica en población general que hayan investigado la prevalencia de psicopatología en tres generaciones. El hallazgo acerca de que la prevalencia de trastornos entre los padres de los adultos entrevistados, $24 \%$, y la de estos últimos, $29 \%$, sea relativamente similar, y de que la prevalencia de probables trastornos entre los menores de 4 a 16 años de estar conviviendo en el mismo hogar, sea similar a la estimada en un estudio anterior respecto a la población 
de 3 a 12 años ${ }^{25}$ en la misma circunstancia es un dato interesante, dado que sugiere una cierta estabilidad de la magnitud del problema en nuestra población. Aunado a este hallazgo, el riesgo general para desarrollar psicopatología obtenido entre los padres e hijos de tres generaciones, 2.5 y 2.7 veces, sugiere también una estabilidad; y en cuanto a la magnitud del riesgo, concuerda con los resultados obtenidos, para trastornos específicos, entre miembros de dos generaciones en la población general de Estados Unidos de América donde el riesgo transmitido fue también considerado como moderado. ${ }^{6}$

Respecto a la importancia de los antecedentes familiares es de interés comparar el riesgo que se ha encontrado en estudios recientes, respecto a otro tipo de enfermedades. Un estudio de casos y controles con base poblacional acerca del cáncer prostático ${ }^{26}$ reportó una razón de momios de 2.7 (IC 95\% 1.5, 4.7), en hispanos en Nuevo México, resultando similar al de la población blanca en el mismo estado: $R M=2.0$ (IC 95\% $1.3,3.1)$. Respecto al lupus eritematoso sistémico en población mexicana, ${ }^{27}$ los antecedentes familiares de la presencia de un trastorno de los tejidos conectivos mostraron una $R M=2.6$ (IC 95\% 1.2, 4.5) utilizando como controles a pacientes sin enfermedades autoinmunes.

A la luz de los resultados anteriores, la estimación del riesgo familiar para desarrollar psicopatología obtenida en el presente estudio adquiere mayor relevancia, resaltando la importancia de la investigación en este campo, y de la evaluación en un nivel de la historia clínica de este tipo de antecedentes.

En el presente estudio la comorbilidad entre diferentes grupos de trastornos se asoció con un mayor riesgo para desarrollar psicopatología. El hallazgo concuerda con los resultados de estudios que han sugerido, respecto a los trastornos psiquiátricos más frecuentes, que existe una predisposición familiar cuya expresión, en términos de trastornos específicos, es variable. Es decir, que la transmisión requiere de la interacción de diversos genes y factores ambientales que transmiten la predisposición a la enfermedad, pero que no siempre conducen a la expresión de un trastorno. Así, diversos trastornos parecen comportarse como grupos antes que como entidades únicas, con factores etiológicos y con factores de riesgo diferentes. ${ }^{8,9}$

Aunque en el presente estudio no se hicieron análisis de segregación, los resultados acerca del riesgo familiar para desarrollar psicopatología, en principio, estarían de acuerdo con la hipótesis de que la transmisión intergeneracional sigue un modelo mixto de herencia, en particular tomando en consideración el espectro de la comorbilidad entre trastornos. ${ }^{7}$ No obstante, es preciso realizar análisis por grupos de trastornos en la infancia y por trastornos específicos.

Limitaciones: el hecho de que la información provenga de un solo informante advierte acerca de posibles sesgos. La validez del método de historia familiar ha sido examinada frente a la de las entrevistas personales habiendo mostrado ser altamente específico, pero con una sensibilidad entre baja a moderada, característica que en lugar de exagerar atenúa la agregación familiar. ${ }^{6,18,19}$ Sin embargo, Chapman y colaboradores ${ }^{28}$ demostraron la sensibilidad de la historia familiar en los informantes "afectados" fue superior a la de los no "afectados", resaltando que no se puede asumir que la información dada por personas que hayan tenido un problema psiquiátrico sea equivalente a la de personas sin ese tipo de problemas, y advirtieron acerca de un sesgo a sobrestimar la agregación del trastorno en el ámbito familiar. Para resolver esta discrepancia, se diseñó un estudio para evaluar la validez predictiva de ambos métodos, es decir el de las entrevistas directas con los familiares y el de la historia familiar, habiendo encontrado que ambos son válidos y, más aún, cada uno aporta información independiente y complementaria acerca de la historia del fenómeno. ${ }^{29}$ De esta manera, se ha concluido que los reportes basados en el método de la historia familiar pueden ser contemplados como otra herramienta de valoración de los trastornos psiquiátricos que posee una utilidad potencial al igual que importantes limitaciones, más que ser un pobre sustituto de entrevistas personales. ${ }^{6}$

De manera similar, ha existido polémica acerca de la confiabilidad y validez de las fuentes de información para realizar un diagnóstico en los niños. Ante este panorama, algunos investigadores han recomendado que toda la información epidemiológica sea considerada como "específica del informante", ${ }^{30,31}$ en otras palabras, la identificación de los diagnósticos y los factores de riesgo se harán de acuerdo con la percepción del informante. Nuestro grupo de investigación se encuentra precisamente desarrollando nuevas investigaciones que permitirán valorar el alcance y limitaciones específicas de estas mediciones, y generar métodos cada vez más válidos y precisos.

En virtud de que en el presente trabajo la información procede únicamente del padre entrevistado y, por lo tanto, aplica la citada observación, es pertinente señalar que en un estudio en el que se siguió por 14 años a una muestra poblacional de niños y adolescentes, ${ }^{32}$ se encontró una estabilidad considerable en el reporte de los padres a lo largo del tiempo.

A pesar de que el estudio únicamente está basado en la información obtenida de los probandos adultos, tiene menos sesgos en cuanto a búsquedas de ayuda, 
referencia y utilización de servicios, en comparación con estudios basados en muestras clínicas. Cabe resaltar su originalidad en cuanto a la información respecto a tres generaciones y su aportación para investigaciones familiares y genéticas acerca de trastornos psiquiátricos que se desarrollan en la actualidad en nuestro país, así como para el enfoque del tratamiento de las personas y de las familias que acuden a los servicios de atención.

Finalmente, en relación con la vigilancia y cuidado de la salud mental a partir de la infancia, se ha señalado que el estudio de los hijos de probandos adultos puede aportar información acerca de: a) la presencia de trastornos y /o de síntomas entre generaciones; $b$ ) signos tempranos y modos de manifestación del trastorno durante la infancia, y c) factores de riesgo o de protección que median en el desarrollo de los trastornos. ${ }^{1}$ Así, análisis subsecuentes del presente estudio podrán aportar datos acerca del riesgo familiar y de factores de riesgo, aclarar la relación entre diferentes categorías diagnósticas. Los hallazgos pueden tener implicaciones para la detección y tratamiento oportunos.

\section{Referencias}

1.W eissman MM, Merikangas KR, John K,W ickramaratne P, Prusoff BA, Kidd KK. Family-genetic studies of psychiatric disorders. Arch Gen Psychiatry 1986; 43:1104-1116.

2. N icolini SH. Bases genéticas de la mente. México, DF: Instituto Mexicano de Psiquiatría; 1999.

3. Costello CG. The similarities and dissimilarities between community and clinic cases of depression. Brit J Psychiatry 1990; 157:812-821.

4. C araveo AJ, Colmenares BE, Saldívar HG. Morbilidad psiquiátrica en la Ciudad de México: prevalencia y comorbilidad en la vida. Salud Mental 1999; 22 (número especial):62-67.

5. W orld Health 0 rganization. International Consortium in Psychiatric Epidemiology. C ross-national comparisons of the prevalence and correlates of mental disorders. Bulletin W orld Health 0 rganization 2000; 78(4):413-426.

6. Kendler K, Davis CG, Kessler RC. The familial aggregation of common psychiatric and substance use disorders in the $\mathrm{N}$ ational Comorbidity Survey: A family history study. Brit J Psychiatry 1997; 170:541-548. 7.Vieland VJ, Susser E,W eissman MM. G enetic epidemiology in psychiatric research. En: Mendlewicz J, Papadimitriou GN Eds. Clinical psychiatry. Genetics of mental disorders. Part I:Theoretical aspects. London: Baillière Tindall; 1995; 19-45.

8. Andrews G, Stewart G, Morris-Yates A, H olt P, Henderson S. Evidence for a general neurotic syndrome. Brit J Psychiatry 1990; 157:6-12. 9. Black DW, N oyes R, Goldstein RB, Blum N.A family study of obsessive-compulsive disorder. Arch Gen Psychiatry 1992; 49:362-368. 10. Cruz C, C amarena B, Mejía JM, Páez F, ErozaV, D e la Fuente JR et al. The dopamine $\mathrm{D} 2$ receptor gene Taq1 A1 polymorphism and alcoholism in a Mexican population. Arch Med Research 1995; 26(4):421-426. 11. C amarena B, Rinetti G, C ruz C, Gómez A, De la Fuente JR, N icolini $H$.Additional evidence that genetic variation of Mao-A gene supports a gender subtype in obsessive-compulsive disorder.Am J Med Genetics 2001; 105(3):279-282.

12. N icolini H, Cruz C, Camarena B, Páez F, De la Fuente JR. Understanding the genetic basis of obsessive-compulsive disorder. CN S Spectrums 1999; 4:32-48.

13. Rineti $G, C$ amarena B, C ruz C, A piquián R, Fresán A, Paéz $F$ et al. Dopamine $D 4$ receptor (DRD 4) gene polymorphism in the first psychotic episode. Arch Med Research 2001; 32:35-38.

14. Caraveo AJ, Martínez N, Rivera E. Un modelo para estudios epidemiológicos sobre la salud mental y la morbilidad psiquiátrica. Salud Mental 1998; 21(1):48-57.

15. C araveo-Anduaga J, C olmenares-Bermúdez E, Martínez-Vélez N A. Síntomas, percepción y demanda de atención en salud mental en niños y adolescentes de la Ciudad de México. Salud Publica Mex 2002; 44(6):492-498.

16. Vega W A, A guilar-G axiola S, Andrade L, Bijil R, Borges G, C araveo J. et al. Prevalence and age of onset for drug use in seven international sites: Results from the International Consortium in Psychiatric Epidemiology . Drug \& Alcohol Dependence 2002; 68:285-297. 17. W orld Health 0 rganization. Composite International Diagnostic Interview. G inebra, Suiza:W orld Health O rganization; 1993.

18. Andreasen NC, Endicott J, Spitzer RL.The family history method using diagnostic criteria: Reliability and validity. Arch Gen Psychiatry 1977; 34:1229-1235.

19. Andreasen N C, Rice J, Endicott J.The family history approach to diagnosis: How useful is it? Arch Gen Psychiatry 1986; 41:421-429. 20. W orld Health 0 rganization. The IC D-10 Classification of mental and behavioural disorders: Diagnostic criteria for research. Ginebra: W HO ; 1993.

21.W enar C, Kenig P. D evelopmental psychopathology. Boston: McG raw-Hill; 2000.

22. Hosmer DW, Lemeshow S.Applied logistic regression. N uevaYork: W iley; 2000.

23. Liang KY, Zeger SL. Longitudinal data analysis using generalized linear models. Biometrika 1986; 73:13-22.

24. Diggle PJ, Liang KY, Zeger SL.A nalysis of longitudinal data. N ueva York: 0 xford University Press; 1995.

25. C araveo AJ, Medina-Mora ME,Villatoro J, López-Lugo EK, Martínez VA. Detección de problemas de salud mental en la infancia. Salud Publica Mex 1995; 37(5):446-451.

26. Stone SN , Hoffman RM, Tollestrup K, Stidley CA, W itter JL, Gilliland FD. Family history, hispanic ethnicity, and prostate cancer risk. Ethnicity \& D isease 2003; 13(2):233-239.

27. Zonana-N acach A, Rodríguez-G uzmán LM, Jiménez-Balderas FJ, Camargo-Coronel A, Escobedo-de la Peña J, Fraga A. Factores de riesgo relacionados con lupus eritematoso sistémico en población mexicana. Salud Publica Mex 2002; 44(3):213-218.

28. Chapman TF, Mannuza S, Klein DF, Fyer AJ. Effects of informant mental disorder on psychiatric family history data.Amer J Psychiatry 1994; 151:574-579.

29. Kendler KS, Roy MA.Validity of a diagnosis of lifetime major depression obtained by personal interviews versus family history. Amer J Psychiatry 1995; 152:1608-1614.

30. Boyle MH, O fford DR, Racine Y, Szatmari P, Fleming JE, Sanford M. Identifying thresholds for classifying childhood psychiatric disorder: Issues and prospects. J Acad Child Adolesc Psychiatry 1996; 35:14401448.

31. 0 fford DR, Boyle MH, RacineY, Szatmori P, Flening JE, Sandford M et al. Integrating assessment data from multiple informants. J A cad C hild Adolesc Psychiatry 1996; 35:1078-1085.

32. Hofstra MB, der Ende JV,Verhulst FC. C ontinuity and change of psychopathology from childhood into adulthood:A 14-year follow-up study. J A cad C hild Adolesc Psychiatry 2000; 39:850-858. 